\title{
Comportamento de galinhas poedeiras em sistema cage free em diferentes idades a a
}

\section{qualidade de ovos}

\author{
Behavior of laying hens in a cage free system at different ages and egg quality \\ Comportamiento de gallinas ponedoras em un sistema sin jaulas a diferentes edades y calidad del \\ huevo
}

Recebido: 12/03/2021 | Revisado: 18/03/2021 | Aceito: 22/03/2021 | Publicado: 30/03/2021

Renata Gimenes Leite

ORCID: https://orcid.org/0000-0001-7924-9084 Universidade Estadual Paulista, Brasil E-mail: renata.gleite@ hotmail.com

Érik dos Santos Harada

ORCID: https://orcid.org/0000-0001-9851-2722 Universidade Estadual Paulista, Brasil E-mail:erik.harada8@gmail.com

Douglas D'Alessandro Salgado

ORCID: https://orcid.org/0000-0002-6729-5128 Universidade Estadual Paulista, Brasil

E-mail:douglas.salgado@unesp.br

Mario Mollo Neto

ORCID: https://orcid.org/0000-0002-8341-4190 Universidade Estadual Paulista, Brasil

E-mail:mario.mollo@unesp.br

Leda Gobbo de Freitas Bueno

ORCID: https://orcid.org/0000-0001-8188-0000

Universidade Estadual Paulista, Brasil

E-mail: leda.bueno@unesp.br

\begin{abstract}
Resumo
A produção de ovos no Brasil tem crescido fator que acarretou um aumento na densidade de animais nas instalações, gerando diminuição da ambiência e do bem-estar. Desta forma este estudo teve como objetivo realizar uma análise em sistemas de criação de poedeiras, tipo cage free sem enriquecimento e cage free com enriquecimento, no pico e final de produção e sua relação com o enriquecimento ambiental e qualidade de ovos. Para o tratamento com enriquecimento foram utilizados: ninho, poleiro, chocalho, fardos de capim nativo e galhos de Cambuci. Enquanto o tratamento sem enriquecimento possuía apenas um ninho, ambos os tratamentos possuíam comedouros do tipo tubular, bebedouros do tipo pendular e cama de palha de arroz. Foram avaliados o comportamento animal e qualidade dos ovos. Na análise de comportamento obtivemos diferença entre os comportamentos de ninho, empoleirar, ciscar, perseguir e coçar cabeça, possivelmente pelo fato das aves utilizarem dos meios que eram fornecidos e poderem expressar seu comportamento natural. Dentro da qualidade de ovos, observamos uma diferença nos parâmetros de coloração da gema, peso, altura de albúmen, unidade Haugh, espessura da casca e índice de gema (YCF), onde o peso e a coloração da gema foram maiores no tratamento sem enriquecimento, podendo ser devido ao maior consumo de alimento, já que as aves nesse tratamento não possuíam outros meios de distração. Conclui-se que o enriquecimento promove alterações positivas tanto na expressão de comportamentos naturais das poedeiras como melhora os principais requisitos em relação à qualidade dos ovos.
\end{abstract}

Palavras-chave: Indicador de bem-estar animal; Densidade animal; Fase de produção; Produtividade.

\begin{abstract}
The production of eggs in Brazil has grown, which has led to an increase in the density of animals in the facilities, generating a decrease in ambience and well-being. Thus, this study aimed to carry out an analysis in laying systems, laying cage free without enrichment and cage free with enrichment, at the peak and end of production and its relationship with environmental enrichment and egg quality. For the treatment with enrichment were used: nest, perch, rattle, bales of native grass and branches of Cambuci. While the treatment without enrichment had only one nest, both treatments had feeders of the tubular type, drinkers of the pendular type and bed of rice straw. Animal behavior and egg quality were evaluated. In the behavior analysis, we obtained a difference between nesting, perching, scratching, chasing and scratching head behaviors, possibly due to the fact that birds use the means that were provided and can express their natural behavior. Within egg quality, we observed a difference in the parameters of yolk color, weight, albumen height, Haugh unit, shell thickness and yolk index (YCF), where yolk weight and color
\end{abstract}


were higher in the treatment without enrichment, which may be due to the greater consumption of food, since the birds in this treatment did not have other means of distraction. It is concluded that enrichment promotes positive changes both in the expression of natural laying behaviors and improves the main requirements in relation to egg quality.

Keywords: Animal welfare indicator; Animal density; Production phase; Productivity.

\section{Resumen}

La producción de huevos en Brasil ha crecido, lo que ha provocado un aumento de la densidad de animales en las instalaciones, generando una disminución del ambiente y el bienestar. Por lo tanto, este estudio tuvo como objetivo realizar un análisis sobre los sistemas de puesta, tipo jaula libre sin enriquecimiento y jaula libre con enriquecimiento, en pico y final de producción y su relación con el enriquecimiento ambiental y la calidad del huevo. Para el tratamiento de enriquecimiento se utilizaron: nido, percha, sonajero, fardos de pasto nativo y ramas de Cambuci. Mientras que el tratamiento sin enriquecimiento tuvo un solo nido, ambos tratamientos contaron con comederos de tipo tubular, bebederos de tipo pendular y lecho de paja de arroz. Se evaluó el comportamiento animal y la calidad del huevo. En el análisis de comportamiento, obtuvimos una diferencia entre los comportamientos de anidar, posarse, rascarse, perseguir y rascarse la cabeza, posiblemente debido al hecho de que las aves utilizan los medios que se les proporcionaron y pueden expresar su comportamiento natural. Dentro de la calidad del huevo, observamos una diferencia en los parámetros de color de la yema, peso, altura de la albúmina, unidad de Haugh, grosor de la cáscara e índice de la yema (YCF), donde el peso y el color de la yema fueron mayores en el tratamiento sin enriquecimiento, lo que puede deberse al mayor consumo de alimento, ya que las aves en este tratamiento no contaban con otros medios de distracción. Se concluye que el enriquecimiento promueve cambios positivos tanto en la expresión de los comportamientos naturales de puesta como mejora los principales requisitos en relación a la calidad del huevo.

Palabras clave: Indicador de bienestar animal; Densidad animal; Etapa de producción; Productividad.

\section{Introdução}

O sistema de criação em gaiolas tornou-se uma das maiores polêmicas acerca do bem-estar animal, por causa do reduzido espaço que é oferecido e a ausência de elementos de enriquecimento que impossibilitam ou limitam o repertório de atividades consideradas importantes para o animal, de modo que sistemas alternativos de criação para poedeiras têm sido cada vez mais estudados (Batista et al, 2012). A origem dos alimentos e como são produzidos, tem sido questionado pelos consumidores. Em relação aos alimentos de origem animal, a preocupação vem da forma como estes são criados e os métodos que são utilizados para a produção destes alimentos. Sendo assim, o bem-estar animal é uma preocupação que acomete grande parte dos consumidores (Ribeiro, 2020). Broom e Molento (2004) afirmam que o bem-estar animal está entre os fatores considerados parte da sustentabilidade e da moralidade dos sistemas de criação de animais. Comportamentos classificados como anômalos e agonísticos nos mostram que essas aves não estão tendo um manejo adequado ou fatores ambientais estão interferindo no seu comportamento (Broom, 1988).

O comportamento de alongar pode ser utilizado para avaliar o bem-estar animal (Li, et al., 2021). Aves em condições de bem-estar expressam por mais tempo comportamentos de banho de poeira, bater asas e alisamento de penas (Bayram \& Özkan, 2010; Rentsch et al, 2019). Comportamentos de alisamento de penas e arrepiar penas são comportamentos sociais e indicadores importantes de bem-estar (Nicol, 1989). Bessei (2006) afirma que a falta de exercícios é a principal causa de fraqueza nas pernas e longos períodos de prostração produzem lesões de peito e pernas, enquanto que Bokkers e Koene (2003) afirmam que poleiros e boa qualidade de cama são cruciais para prevenir anomalias físicas em frangos de corte.

Aves adultas criadas em aviários fechados tem dificuldade de se adaptar em ambientes enriquecidos (Bari et al, 2020; Maclachlan et al, 2020). Porém, o comportamento de uso do ninho de caixa não é afetado pela experiência anterior da ave, sendo que aves de gaiolas apresentaram mesma intensidade de uso dos ninhos que galinhas já criadas em ambientes enriquecidos (Cooper \& Appleby, 1995). O comportamento de uso de ninho tem se mostrado muito importante, pois as aves deixam de se alimentar e de expressar outros comportamentos como banho de poeira e se arrumar para permanecerem no ninho (Engel et al, 2019). Os ninhos tipo caixa são importantes para que outros comportamentos naturais sejam expressos, sendo assim uma alternativa para minimizar o estresse causado pela produção convencional de ovos (Becker, 2005). Bari et al. (2020) 
verificaram que mudanças semanais no enriquecimento ambiental de poedeiras favoreceu ao grupo de aves a botarem mais em ninhos tipo caixa. O enriquecimento ambiental tem o objetivo de estimular comportamentos naturais e melhorar o bem-estar das aves (Ali et al, 2016). As poedeiras intensificam o uso de poleiros durante o período noturno (Liu et al, 2018) e preferem poleiros mais altos (Brendler \& Schrader, 2016), indicando que a altura do poleiro é característica importante as ser considerada para a sua disponibilização. Ali et al. (2016), Faure e Jone (1982) verificaram que a linhagem da poedeira afeta o uso de poleiros, sendo que galinhas de linhagem brancas empoleiraram mais que galinhas de linhagem marrom.

Com a proibição do uso de gaiolas pela União Europeia, foram necessárias algumas alternativas no modo de criação, com isso surgiram as gaiolas enriquecidas, cage free, sistema free range, aviary e sistemas barn (Onbasilar et al, 2015). A qualidade da produção é um dos principais interesses dos produtores e consumidores de ovos, uma vez que está diretamente relacionada a fatores como: higiene, sanidade e principalmente a saúde e bem-estar dos animais (Barbosa Filho et al, 2007). Análises de parâmetros produtivos e da qualidade dos ovos são exemplos de algumas medidas adotadas para determinação dos efeitos do ambiente de criação sobre o desempenho e o bem-estar das aves (Alves et al, 2007). Neste contexto, a produção, a produtividade e a qualidade do produto são os principais interesses dos produtores e consumidores de ovos uma vez que está diretamente relacionada a fatores, como higiene, sanidade e, principalmente, à saúde e ao bem-estar dos animais (Trindade et al, 2007).

Assim, o objetivo deste trabalho é realizar uma análise comportamental e de qualidade de ovos em sistemas de criação de poedeiras, tipo cage free, no pico e final de produção e sua relação com o enriquecimento ambiental.

\section{Metodologia}

\subsection{Localização do Experimento e condições climáticas}

O experimento foi conduzido no setor de avicultura nas dependências da Faculdade de Ciências Agrárias e Tecnológicas - UNESP, Campus de Dracena, com dimensões de 23 × 8,3 x 3m (comprimento, largura e pé-direito) e foi adaptado para receber as poedeiras. O aviário é climatizado por pressão positiva, com o uso de ventiladores e aspersão. Sua localização geográfica é latitude de $21^{\circ} 29 \mathrm{~min} \mathrm{~S}$, longitude de $51^{\circ} 52 \mathrm{~min} \mathrm{~W}$ e altitude de $421 \mathrm{~m}$ e sua orientação é leste-oeste. O clima da região, segundo KOPPEN-GEISER é subtropical Aw (invernos secos seguidos de verões muito quentes).

O experimento foi realizado em duas etapas (ciclos) de 21 dias cada, sendo o primeiro entre os meses de setembro/outubro do ano de 2018 (estação de primavera) e o segundo ciclo em dezembro do ano de 2019 (estação de verão). Para ambos os ciclos se realizou um período de adaptação das aves de 7 dias.

Foi imposto, para ambas as etapas, um manejo de restrição alimentar, de 100g/animal colocado uma vez ao dia no período da manhã. A água foi ad libitum e os bebedouros higienizados semanalmente. As camas foram revolvidas toda semana. O sistema de luz adotado foi de 11 horas de luz natural complementada por 4 horas de iluminação artificial (lâmpadas tipo LED com potência de 160 watts).

O ambiente externo ao aviário foi monitorado e registrado por uma estação meteorológica situada na própria Faculdade de Ciências Agrárias e Tecnológicas - UNESP, Campus de Dracena. Já no ambiente interno foram registradas as temperaturas de bulbo seco (Tbs), temperatura de globo negro (Tgn) e umidade relativa do ar (UR), utilizando 3 dataloggers HOBO ${ }^{\circledR}$ U12-012 com canal externo para sensor de temperatura TMC50-HD. As coletas foram realizadas a cada trinta minutos seguindo metodologia adaptada de Pereira (2007) e Silva et al, (2013).

\subsection{Projeto experimental, Aves e Descrição dos tratamentos}

Foram utilizadas 60 galinhas poedeiras da linhagem Hysex White ${ }^{\circledR}$ para cada etapa (ciclo). As aves foram selecionadas aleatoriamente, provindas do mesmo lote de criação, tanto para a etapa 1 quanto para a etapa 2 . Assim, para o 
primeiro ciclo (etapa 1), as aves tinham 25 semanas de idade e no segundo ciclo (etapa 2) as mesmas tinham e média 60 semanas de idade.

As aves foram alojadas em aviário o qual é dividido em boxes, sendo distribuídos lateralmente. Cada box possui $3 \mathrm{~m}^{2}$ de área $(1,2 \times 2,50 \mathrm{~m})$. Os boxes foram divididos em 2 sistemas, sendo um denominado "sem enriquecimento" e o segundo "com enriquecimento".

Tanto no sistema "sem enriquecimento" como no sistema "com enriquecimento" para cada box, alocou-se 1 ninho de madeira tipo pinus de reflorestamento (Pinus elliottii) com dimensão de 40 x $40 \mathrm{~cm}, 1$ comedouro tipo tubular da cor vermelha e 1 bebedouro tipo pendular da cor vermelha. Os boxes eram forrados com cama de palha de arroz de primeiro uso.

O sistema "enriquecido" diferencia-se pela inserção, por box, de 1 poleiro de madeira tipo pinus de reflorestamento (P. elliottii) com medidas de $120 \mathrm{~cm}$ de comprimento e $3,5 \mathrm{~cm}$ de diâmetro (fixados transversalmente entre uma parede e outra no canto anterior (próximo a porta de entrada do box) a $50 \mathrm{~cm}$ do piso, 1 chocalho feito de material de reciclável de plástico (Poli(Tereftalato de Etileno)) da cor verde; fardos de capim nativo tipo Aristida jubata (Nassella melanosperma) e galhos de Cambuci (Campomanesia phaea).

\subsection{Qualidade do ovo}

Para cada etapa do experimento, nos últimos 3 dias de cada ciclo, todos os ovos produzidos foram coletados para a análise de qualidade. Estes ovos foram armazenados em temperatura de resfriamento $\left(5^{\circ} \mathrm{C}\right)$ por 72 horas. Para a análise de qualidade utilizou-se a máquina modelo DET 6000 da fabricante $\mathrm{NABEL}^{\circledR}$, a qual fornece valores para peso do ovo, resistência de casca, espessura da casca, altura da gema, coloração da gema e Unidade Haugh.

\subsection{Comportamentos monitorados}

A ocorrência (número de vezes que qualquer ave e em cada box, expressou um comportamento específico) e duração (em segundos) de cada comportamento foram quantificados. Comportamentos foram observados, in loco, por um mesmo observador, seguindo metodologia adaptada de Tavares et al. (2015), por 15 minutos, nos 21 dias de cada ciclo, 3 vezes ao dia (07:00 - 07:15h, 14:00-14:15h e 19:00h-19:15h). Um mesmo observador durante as duas etapas dos experimentos realizou a coleta dos dados visualmente.

Os comportamentos analisados foram: arrumar penas, banho de poeira, bater as asas, beber água, bicar, ciscar, coçar cabeça, comer, empoleirar, esticar perna, uso do ninho, perseguir e sentar.

\subsection{Análise Estatística}

As verificações foram feitas para dados provenientes de duas condições diferentes: aves alocadas com densidade de 3 aves/box e aves alocadas com densidade de 6 aves/box.

Os testes estatísticos foram produzidos em função dos tipos de variáveis analisados e dos objetivos propostos. Os procedimentos foram conduzidos no software MINITAB $19^{\circledR}$.

\subsubsection{Comportamento}

Para verificar se a presença de enriquecimento ambiental promove alterações significativas nas taxas de ocorrência de diversos comportamentos, foram executados testes de hipótese para diferença em taxas (testes de Poisson para 2 amostras), com nível de significância de 5\%. O comprimento da observação para a execução do teste é definido pelo produto da quantidade de aves no box e do período de observação (em minutos). 


\subsubsection{Qualidade dos ovos}

A fim de comparar as médias de diferentes parâmetros quantitativos de qualidade do ovo, foram executados testes de hipóteses para diferença entre médias (testes $\mathrm{T}$ para 2 amostras), com nível de significância de 5\%. Já para a variável qualitativa coloração superior de gema (presença ou ausência), foram realizados testes $\chi^{2}$ com nível de significância de 5\%.

\section{Resultados e Discussão}

\subsection{Comportamento}

No primeiro experimento, delineado com densidade de 3 aves por box, o Teste de hipótese para diferença nas taxas de comportamento $(\alpha=0,05)$ detectou diferenças entre as taxas de ocorrência dos comportamentos coçar a cabeça $(\mathrm{p}=0,023)$, empoleirar $(\mathrm{p}=0,000)$ e ninho $(\mathrm{p}=0,000)$, como mostrado na Tabela 1 .

Tabela 1 - Resultados do Teste de hipótese para diferença nas taxas de comportamento nos dois tratamentos para $\rho=3$ aves/box

\begin{tabular}{|c|c|c|c|c|}
\hline Comportamento & Tratamento & Frequência & Taxa $^{¥}$ & P-valor ${ }^{\S}$ \\
\hline \multirow{2}{*}{ Arrumar penas } & Com enriquecimento & 47 & 0,0514 & \multirow[b]{2}{*}{0,325} \\
\hline & Sem enriquecimento & 59 & 0,0634 & \\
\hline \multirow{2}{*}{ Banho de poeira } & Com enriquecimento & 14 & 0,0153 & \multirow{2}{*}{0,107} \\
\hline & Sem enriquecimento & 6 & 0,0065 & \\
\hline \multirow{2}{*}{ Bater as asas } & Com enriquecimento & 1 & 0,0011 & \multirow{2}{*}{1,000} \\
\hline & Sem enriquecimento & 2 & 0,0022 & \\
\hline \multirow[b]{2}{*}{ Beber água } & Com enriquecimento & 26 & 0,0284 & \multirow[b]{2}{*}{0,473} \\
\hline & Sem enriquecimento & 33 & 0,0355 & \\
\hline \multirow{2}{*}{ Bicar } & Com enriquecimento & 36 & 0,0393 & \multirow{2}{*}{0,138} \\
\hline & Sem enriquecimento & 24 & 0,0258 & \\
\hline \multirow[b]{2}{*}{ Ciscar } & Com enriquecimento & 91 & 0,0995 & \multirow{2}{*}{0,121} \\
\hline & Sem enriquecimento & 116 & 0,1247 & \\
\hline \multirow[b]{2}{*}{ Coçar cabeça } & Com enriquecimento & 3 & 0,0033 & \multirow[b]{2}{*}{$\mathbf{0 , 0 2 3}$} \\
\hline & Sem enriquecimento & 13 & 0,0140 & \\
\hline \multirow[b]{2}{*}{ Comer } & Com enriquecimento & 122 & 0,1333 & \multirow{2}{*}{0,950} \\
\hline & Sem enriquecimento & 126 & 0,1355 & \\
\hline \multirow[b]{2}{*}{ Empoleirar } & Com enriquecimento & 68 & 0,0743 & \multirow{2}{*}{0,000} \\
\hline & Sem enriquecimento & 0 & 0,0000 & \\
\hline \multirow{2}{*}{ Esticar perna } & Com enriquecimento & 6 & 0,0066 & \multirow{2}{*}{0,492} \\
\hline & Sem enriquecimento & 3 & 0,0032 & \\
\hline Ninho & Com enriquecimento & 11 & 0,0120 & 0,000 \\
\hline
\end{tabular}


Research, Society and Development, v. 10, n. 4, e6010413833, 2021

(CC BY 4.0) | ISSN 2525-3409 | DOI: http://dx.doi.org/10.33448/rsd-v10i4.13833

\begin{tabular}{cccc}
\hline & & & \\
\hline & Sem enriquecimento & 42 & 0,0452 \\
\hline \multirow{2}{*}{ Perseguir } & Com enriquecimento & 4 & 0,0044 \\
& Sem enriquecimento & 5 & 0,0054 \\
\cline { 2 - 4 } Sentar & Com enriquecimento & 8 & 0,0087 \\
\cline { 2 - 4 } & Sem enriquecimento & 5 & 0,0054 \\
\hline
\end{tabular}

¥Taxa de ocorrência do comportamento, medida em ocorrências/minuto/ave

${ }^{\S}$ Obtido por meio do Teste de hipótese para diferença nas taxas para o método exato (Nível de Confiança: $95 \%$ ). As diferenças têm significância estatística quando $\mathrm{P}$-valor $<0,05$.

$\rho$ : Densidade

Fonte: Autores.

A taxa de ocorrência do comportamento coçar a cabeça foi maior no tratamento sem enriquecimento $(0,0140$ ocorrências/minuto/ave) do que no tratamento enriquecido ( 0,0033 ocorrências/minuto/ave). Convertendo a unidade das taxas de minuto para horas, a magnitude da diferença fica mais evidente: sem enriquecimento ( 0,84 ocorrências/hora/ave), em contraste com $(0,20$ ocorrências/hora/ave) no tratamento enriquecido. O comportamento empoleirar teve maior taxa de ocorrência no tratamento enriquecido (4,46 ocorrências/hora/ave) do que no tratamento sem enriquecimento (0,13 ocorrências/hora/ave), isso pode ter acontecido devido à presença do poleiro que é um elemento atrativo para as aves e que remete ao comportamento natural da espécie. Para o comportamento ninho, cujos resultados também apresentam diferença estatística significativa, o tratamento sem enriquecimento apresentou maior taxa de ocorrência (0,72 ocorrências/hora/ave), contra (2,71 ocorrências/hora/ave) no tratamento com enriquecimento. Pensando novamente como uma forma de atrativo, as aves do tratamento enriquecido possuíam outros meios de distração além do ninho.

Com os resultados do teste de Poisson, não foram encontradas diferenças estatísticas significativas $(\alpha=0,05)$ entre as taxas de ocorrência dos demais comportamentos.

Na segunda parte do experimento (repetição), delineado com densidade de 6 aves/box, foram detectadas diferenças significativas $(\alpha=0,05)$ para os comportamentos: ciscar $(\mathrm{p}=0,000)$, empoleirar $(\mathrm{p}=0,000)$ e perseguir $(\mathrm{p}=0,035)$, como mostrado na Tabela 2. 
Tabela 2 - Resultados do teste de hipótese para diferenças nas taxas de comportamento nos dois tratamentos para $\rho=6$ aves/box.

\begin{tabular}{|c|c|c|c|c|}
\hline Comportamento & Tratamento & Frequência & $\operatorname{Taxa}^{¥}$ & P-valor ${ }^{\S}$ \\
\hline \multirow{2}{*}{ Arrumar penas } & Com enriquecimento & 22 & 0,0509 & \multirow{2}{*}{0,771} \\
\hline & Sem enriquecimento & 25 & 0,0579 & \\
\hline \multirow{2}{*}{ Banho de cama } & Com enriquecimento & 4 & 0,0093 & \multirow{2}{*}{0,118} \\
\hline & Sem enriquecimento & 11 & 0,0255 & \\
\hline \multirow{2}{*}{ Bater as asas } & Com enriquecimento & 8 & 0,0185 & \multirow{2}{*}{1,000} \\
\hline & Sem enriquecimento & 8 & 0,0185 & \\
\hline \multirow{2}{*}{ Beber água } & Com enriquecimento & 13 & 0,0301 & \multirow{2}{*}{0,175} \\
\hline & Sem enriquecimento & 22 & 0,0509 & \\
\hline \multirow{2}{*}{ Bicar } & Com enriquecimento & 3 & 0,0069 & \multirow{2}{*}{1,000} \\
\hline & Sem enriquecimento & 3 & 0,0069 & \\
\hline \multirow{2}{*}{ Ciscar } & Com enriquecimento & 23 & 0,0532 & \multirow{2}{*}{0,000} \\
\hline & Sem enriquecimento & 58 & 0,1343 & \\
\hline \multirow{2}{*}{ Coçar cabeça } & Com enriquecimento & 2 & 0,0046 & \multirow{2}{*}{1,000} \\
\hline & Sem enriquecimento & 3 & 0,0069 & \\
\hline \multirow{2}{*}{ Comer } & Com enriquecimento & 53 & 0,1227 & \multirow{2}{*}{0,775} \\
\hline & Sem enriquecimento & 57 & 0,1319 & \\
\hline \multirow{2}{*}{ Empoleirar } & Com enriquecimento & 60 & 0,1389 & \multirow{2}{*}{0,000} \\
\hline & Sem enriquecimento & 0 & 0,0000 & \\
\hline \multirow[b]{2}{*}{ Esticar perna } & Com enriquecimento & 3 & 0,0069 & \multirow[b]{2}{*}{1,000} \\
\hline & Sem enriquecimento & 2 & 0,0046 & \\
\hline \multirow{2}{*}{ Ninho } & Com enriquecimento & 20 & 0,0463 & \multirow{2}{*}{0,871} \\
\hline & Sem enriquecimento & 18 & 0,0417 & \\
\hline \multirow[b]{2}{*}{ Perseguir } & Com enriquecimento & 3 & 0,0069 & \multirow[b]{2}{*}{$\mathbf{0 , 0 3 5}$} \\
\hline & Sem enriquecimento & 12 & 0,0278 & \\
\hline \multirow{2}{*}{ Sentar } & Com enriquecimento & 0 & 0,0000 & \\
\hline & Sem enriquecimento & 0 & 0,0000 & \\
\hline
\end{tabular}

${ }^{\ddagger}$ Taxa de ocorrência do comportamento, medida em ocorrências/minuto/ave

${ }^{\S}$ Obtido por meio do Teste de hipótese para diferença nas taxas para o método exato (Nível de Confiança: 95\%). As diferenças têm significância estatística quando P-valor $<0,05$.

$\rho$ : Densidade

Fonte: Autores. 
A taxa de ocorrência do comportamento ciscar foi maior no tratamento sem enriquecimento, com 0,1343 ocorrências/minuto/ave em oposição a 0,0532 ocorrências/minutos/ave do outro tratamento. O mesmo foi constatado para o comportamento perseguir, que apresentou taxa de 0,0278 ocorrências/minuto/ave no tratamento sem enriquecimento e taxa de 0,0069 ocorrências/minuto/ave no tratamento com enriquecimento, representando um valor de magnitude 4 vezes menor. Ambos os comportamentos podem ter sido evidenciados mais no tratamento sem enriquecimento, pois as aves não tinham outros meios de distração e o aumento na densidade aves/baia pode ter causado esse aumento no comportamento de perseguir.

Já para o comportamento empoleirar, a taxa de ocorrência foi maior no tratamento com enriquecimento $(0,1389$ ocorrências/minuto/ave), não houve ocorrências desse comportamento no tratamento sem enriquecimento, visto que este tratamento não possuía poleiro.

O comportamento sentar não foi observado em nenhum dos tratamentos durante o período de observação, impossibilitando a execução do teste de diferença entre taxas. Para os demais comportamentos, não foram encontradas diferenças estatísticas significativas $(\alpha=0,05)$ entre as taxas de ocorrência com o teste de hipóteses de Poisson.

A complexidade ambiental limitada e o confinamento em gaiolas convencionais restringem fisicamente as galinhas e eliminam muitos de seus comportamentos naturais, como nidificar, empoleirar e coçar (Nicol, 1987; Baxter, 1994). Com a realização do experimento foi possível observar, em ambos os ciclos, que as aves conseguiram demonstrar alguns dos seus comportamentos naturais, apesar dessas aves serem originárias de gaiolas, a alta evolução nos setores da avicultura de postura e o grande avanço da genética nas linhagens comerciais de aves cada vez mais produtivas estarem fazendo com que as linhagens "percam" seus princípios normais de comportamento animal, devido aos avanços genéticos as aves se "esqueceram" de que seu comportamento natural seria botar no ninho (Barbosa Filho, 2004). Guo et al. (2012), observaram que poedeiras criadas em grupos com 48 indivíduos empoleiravam mais e ficavam menos tempo no ninho do que poedeiras criadas em grupos de 21 indivíduos, entretanto, à densidade maior, o menor número de visitas ao ninho e o aumento das frequências de empoleirar e de ciscar indicam que, nessa densidade, há maior concorrência pelos diferentes espaços proporcionados pelo enriquecimento ambiental.

Aves criadas em gaiolas convencionais têm restrita capacidade de circulação e expressão de seus comportamentos naturais, o que compromete sua saúde, bem-estar e produção (Alves et al, 2007). Além disso, a densidade das gaiolas também influencia no comportamento animal. A diminuição da densidade, ou o uso de grupos menores, é benéfico para a colocação de ovos, desempenho e bem-estar, porém o engaiolamento causa uma frustração nos animais (Singer, 2013). Os comportamentos que indicam frustrações são: bicadas severas e bater de asas incessante (Pereira et al, 2013). É esperado que, em grupos maiores, comportamentos agressivos sejam menos frequentes (D'Eath, 1999; Rodenburg et al, 2005).

A incidência de bicadas agressivas foi observada em grupos com maior número de animais (Bilcík et al, 2000). Concordando com os resultados obtidos no presente experimento, no comportamento "perseguir". De acordo com Keeling, (1995), a disputa por recursos e a relação hierárquica são os principais fatores que influenciam o comportamento de poedeiras. D'eath, (1999) e Rodenburg et al. (2005) observaram menor frequência de comportamentos agressivos em grupos maiores, pelo fato de a hierarquia nesses grupos ser baseada em uma avaliação direta de tamanho e outros sinais físicos das aves, e pelo reconhecimento individual, como ocorre em pequenos grupos.

\subsection{Qualidade dos ovos}

A fim de comparar as médias de diferentes parâmetros quantitativos de qualidade do ovo, foram executados testes de hipóteses para diferença entre médias (testes $\mathrm{T}$ para 2 amostras), com nível de significância de 5\%. Já para a variável qualitativa coloração superior de gema (presença ou ausência), foram realizados testes $\chi^{2}$ com nível de significância de 5\%. 
No primeiro experimento o Teste de Hipóteses de Diferença entre Médias para Duas Amostras detectou diferenças para as médias de peso (g) dos ovos provenientes dos dois tratamentos, sendo o ovo do tratamento com enriquecimento mais pesado $(\mathrm{p}=0,045)$. Para a altura do albúmen $(\mathrm{mm})$, tendo o tratamento com enriquecimento produzido maior altura de albúmen $(\mathrm{p}=0,000), 8,107 \mathrm{~mm}$ contra $7,270 \mathrm{~mm}$ do tratamento sem enriquecimento.

A Unidade Haugh $(\mathrm{UH})$ também apresentou diferença significativa $(\mathrm{p}=0,000)$, tendo o tratamento com enriquecimento apresentado o valor médio de 91,23 e o sem enriquecimento de 85,30. A espessura da casca (mm) também apresentou diferença significativa para os dois tratamentos $(\mathrm{p}=0,011), 0,4289 \mathrm{~mm}$ contra $0,4014 \mathrm{~mm}$.

Com os resultados desse teste, não foram encontradas diferenças significativas ao nível de significância de 5\% entre as estimativas dos demais parâmetros de qualidade como presente na Tabela 3.

Tabela 3 - Estimativas de parâmetros de qualidade de ovos oriundos de tratamento com e sem enriquecimento ambiental $(\rho=3$ aves/box).

\begin{tabular}{|c|c|c|c|c|}
\hline Parâmetro & Tratamento & Média & $\mathbf{T}$ & p-valor ${ }^{\S}$ \\
\hline \multirow{2}{*}{$\begin{array}{c}\text { Peso } \\
(\mathrm{g})\end{array}$} & Com enriquecimento & 54,94 & \multirow{2}{*}{$-2,02$} & \multirow{2}{*}{0,045} \\
\hline & Sem enriquecimento & 56,50 & & \\
\hline \multirow{2}{*}{$\begin{array}{l}\text { Altura de gema } \\
\qquad(\mathbf{m m})\end{array}$} & Com enriquecimento & 8,107 & \multirow{2}{*}{3,71} & \multirow{2}{*}{0,000} \\
\hline & Sem enriquecimento & 7,270 & & \\
\hline \multirow{2}{*}{$\begin{array}{l}\text { Índice de gema } \\
\text { (YCF) }\end{array}$} & Com enriquecimento & 5,558 & \multirow{2}{*}{$-1,49$} & \multirow{2}{*}{0,139} \\
\hline & Sem enriquecimento & 5,688 & & \\
\hline \multirow{2}{*}{$\begin{array}{l}\text { Unidade Haugh } \\
\text { (HU) }\end{array}$} & Com enriquecimento & 91,23 & \multirow{2}{*}{3,82} & \multirow{2}{*}{$\mathbf{0 , 0 0 0}$} \\
\hline & Sem enriquecimento & 85,30 & & \\
\hline \multirow{2}{*}{$\begin{array}{c}\text { Resistência da casca } \\
\text { (kgf) }\end{array}$} & Com enriquecimento & 3,99 & \multirow{2}{*}{$-0,86$} & \multirow{2}{*}{0,389} \\
\hline & Sem enriquecimento & 4,18 & & \\
\hline \multirow{2}{*}{$\begin{array}{c}\text { Espessura da casca } \\
\qquad(\mathrm{mm})\end{array}$} & Com enriquecimento & 0,4289 & \multirow{2}{*}{2,57} & \multirow{2}{*}{0,011} \\
\hline & Sem enriquecimento & 0,4014 & & \\
\hline
\end{tabular}

${ }^{\S}$ Obtido por meio do Teste de Hipóteses para Duas Amostras (Confiança 95\%). A diferença entre as médias tem significância estatística quando P-valor < 0,05. $\rho$ : Densidade

Fonte: Autores.

Para o segundo ciclo do experimento, com densidade de 6 aves/box, o Teste de Hipóteses detectou diferença para o Índice da Gema (YCF), com maior valor para o tratamento com enriquecimento $(4,764$ contra 4,234). Não foram encontradas diferenças significativas $(\alpha=0,05)$ entre as estimativas dos demais parâmetros de qualidade como mostra a Tabela 4. 
Tabela 4 - Estimativas de parâmetros de qualidade de ovos oriundos de tratamento com e sem enriquecimento ambiental $(\rho=6$ aves/box).

\begin{tabular}{|c|c|c|c|c|}
\hline Parâmetro & Tratamento & Média & $\mathbf{T}$ & p-valor ${ }^{\S}$ \\
\hline \multirow{2}{*}{$\begin{array}{c}\text { Peso } \\
(\mathrm{g})\end{array}$} & Com enriquecimento & 63,83 & \multirow{2}{*}{0,68} & \multirow{2}{*}{0,499} \\
\hline & Sem enriquecimento & 62,78 & & \\
\hline \multirow{2}{*}{$\begin{array}{l}\text { Altura de gema } \\
\qquad(\mathrm{mm})\end{array}$} & Com enriquecimento & 4,11 & \multirow{2}{*}{$-0,36$} & \multirow{2}{*}{0,717} \\
\hline & Sem enriquecimento & 4,23 & & \\
\hline \multirow{2}{*}{$\begin{array}{l}\text { Índice de gema } \\
\text { (YCF) }\end{array}$} & Com enriquecimento & 4,764 & \multirow{2}{*}{2,85} & \multirow{2}{*}{0,006} \\
\hline & Sem enriquecimento & 4,234 & & \\
\hline \multirow{2}{*}{$\begin{array}{c}\text { Unidade Haugh } \\
\text { (HU) }\end{array}$} & Com enriquecimento & 56,2 & \multirow{2}{*}{$-0,57$} & \multirow{2}{*}{0,570} \\
\hline & Sem enriquecimento & 58,0 & & \\
\hline \multirow{2}{*}{$\begin{array}{c}\text { Resistência da casca } \\
\text { (kgf) }\end{array}$} & Com enriquecimento & 2,70 & \multirow{2}{*}{$-0,18$} & \multirow{2}{*}{0,862} \\
\hline & Sem enriquecimento & 2,75 & & \\
\hline \multirow{2}{*}{$\begin{array}{l}\text { Espessura da casca } \\
\qquad(\mathbf{m m})\end{array}$} & Com enriquecimento & 0,0677 & \multirow{2}{*}{$-0,05$} & \multirow{2}{*}{0,962} \\
\hline & Sem enriquecimento & 0,0726 & & \\
\hline
\end{tabular}

${ }^{\S}$ Obtido por meio do Teste de Hipóteses para Duas Amostras (Confiança 95\%). A diferença entre as médias tem significância estatística quando P-valor $<0,05$.

$\rho$ : Densidade

Fonte: Autores

A associação entre a presença de enriquecimento ambiental e a ocorrência de gemas com coloração superior também foi comparada para os dois experimentos. No primeiro experimento, foi detectada associação significativa $(\mathbf{p}=\mathbf{0 , 0 1 8})$ entre a coloração superior de gema e a presença de enriquecimento ambiental. No segundo experimento, com densidade de 6 aves/box, 17,56\% do total de ovos apresentaram coloração superior de gema. A presença dessa característica não está associada à presença ou à ausência de enriquecimento ambiental, descrito na Tabela 5.

Tabela 5 - Comparação entre ocorrências de ovos com coloração superior de gema para os dois tratamentos, em cada um dos experimentos com diferentes densidades de aves/box.

\begin{tabular}{|c|c|c|c|c|}
\hline $\begin{array}{l}\text { Densidade } \\
\text { (aves/box) }\end{array}$ & Total de ovos & Tratamento & Ocorrências & p-valor ${ }^{\S}$ \\
\hline \multirow{2}{*}{3} & \multirow{2}{*}{121} & Com enriquecimento & 57 & \multirow{2}{*}{0,018} \\
\hline & & Sem enriquecimento & 58 & \\
\hline \multirow{2}{*}{6} & \multirow{2}{*}{63} & Com enriquecimento & 4 & \multirow{2}{*}{0,553} \\
\hline & & Sem enriquecimento & 7 & \\
\hline
\end{tabular}

${ }^{\S}$ Obtido por meio do Teste Qui-Quadrado. A ocorrência de ovos com coloração superior de gema está associada ao tratamento quando P-valor < 0,05 Fonte: Autores. 
Para os produtores, a qualidade do ovo está relacionada com o seu peso e a resistência da casca (como defeitos, sujeiras, quebras e manchas de sangue). Para os consumidores a qualidade está relacionada com o prazo de validade do produto e com as características sensoriais, como cor da gema e da casca. Já para os processadores, a qualidade está relacionada com a facilidade de retirar a casca, com a separação da gema da clara, com as propriedades funcionais e com a cor da gema (especialmente para massas e produtos de padaria) (Rossi \& Pompei, 1995).

Existem alguns métodos para estimar a qualidade de ovos abertos, com bases quantitativas, relacionadas ao albúmen: altura da clara (Wilgus \& Wagenen, 1936); índice do albúmen (Heiman \& Carver, 1936); índice da área do albúmen (Parsons \& Mink, 1937); percentagem da clara espessa e fina (Holts \& Almiquist, 1932); e a unidade "Haugh" (Haugh, 1937). O parâmetro mais usado para expressar a qualidade do albume é a unidade "Haugh".

A unidade "Haugh" é uma expressão matemática que correlaciona o peso do ovo com a altura da clara espessa. De modo geral, quanto maior o valor da unidade "Haugh", melhor a qualidade do ovo (Rodrigues, 1975). O valor da unidade "Haugh" de ovos frescos diminui com o aumento da idade da galinha poedeira (Cunningham et al, 1960; Fletcher et al, 1983). Com o envelhecimento da galinha, ocorre aumento no tamanho dos ovos (Eisen et al, 1962). A composição da ração e a raça da galinha podem afetar o escore da unidade "Haugh". Outros fatores, como estação do ano (Cunningham et al, 1960) e método de criação (Proudfoot, 1962) não parecem afetar o escore da unidade "Haugh". Para Rossi \& Pompei, (1995) o tipo de criação e a estação do ano afetam a composição e a estrutura dos ovos de galinha.

A diminuição nos valores da unidade "Haugh" representa declínio na qualidade do ovo. Segundo Cherian et al. (1990), quando os ovos são armazenados por longos períodos pode ocorrer também a redução do peso do ovo devido à perda de água e a centralização da gema. Fletcher et al. (1983) e Belyavin, (1988) afirmaram que a unidade "Haugh" diminui com o aumento da idade.

Estudos mostram que a quantidade de ovos sujos é superior em sistemas alternativos de produção, pois há contaminação desses ovos na caixa de ninhos ou depósito de ovos em caixas de areia (Vits et al, 2005). Porém quando se fala da produção, Onbasilar et al. (2015), diz que há uma maior produção em gaiolas enriquecidas do que em gaiolas convencionais. Segundo Dutra (2021), o tempo que os ovos permanecem nos ninhos, entre a postura e a coleta, exerce uma influencia negativa sobre as características de qualidade interna do ovo, demandando um desenvolvimento de novas técnicas de coleta e adoção de boas práticas de manejo.

Em relação à densidade de criação Cunningham e Ostrander (1982) e Benyi et al. (2006) afirmam que densidades menores, ou seja, grupo menor de aves por gaiola, produzem ovos mais pesados quando relacionados a grupos de alta densidade. Já de acordo com Lee, (1989), não há diferença entre o peso dos ovos para diferentes densidades de gaiola.

Com relação à espessura da casca, estudos mostram que a casca foi mais espessa em ovos de sistema de criação alternativos do que em gaiolas convencionais (Pavlovski et al, 2001; Hidalgo et al, 2008). Outrora, outros autores observaram casca mais espessa para gaiolas convencionais (Tumova, 2003; Ahammed et al, 2014). Já com relação à força de quebra, de acordo com Casiraghi et al. (2005) há uma correlação indireta entre o tamanho do ovo e a força de quebra.

Singh et al. (2009), relatam que encontraram altura de albúmen maior em sistemas alternativos quando comparados aos convencionais, concordando com o que foi obtido no primeiro ciclo do experimento.

Provavelmente a não diferença encontrada no segundo ciclo do experimento pode ser relacionada ao balanceamento da ração, ou seja, a alimentação não influenciou na qualidade dos ovos.

Analisando a cor da gema, observa-se que os ovos produzidos pelas aves no tratamento enriquecido, do primeiro ciclo, obtiveram uma melhor coloração (AA), possivelmente pelo fato das aves estarem selecionando o alimento. A intensidade de coloração da gema é um assunto de importância prática para Indústria de processamento de ovos, pois a cor é o primeiro critério utilizado na aceitação ou não do consumidor (Silva, 2000). Segundo o mesmo autor, a cor amarelada, característica das 
gemas, é proveniente da absorção dos pigmentos carotenoides presente na ração. Mas, quando são utilizados ingredientes deficientes em xantofilas como o sorgo, mandioca e quirera de arroz, pigmentos devem ser incluídos na ração para melhorar a coloração da gema do ovo.

\section{Conclusão}

Conclui-se que o enriquecimento promove alterações positivas na expressão de comportamentos naturais das poedeiras, como, empoleirar, utilizar o ninho e ciscar e também promoveu uma melhora nos requisitos de qualidade dos ovos, peso dos ovos, unidade Haugh e espessura da casca de ovos no pico de produção e na fase final. Parâmetros que são muito importantes para a qualidade dos ovos e aceitação dos consumidores.

Para futuros estudos é necessário um maior tempo de adaptação das aves provindas de gaiolas, ao sistema cage free, por isso torna-se importante estudos que visem criar linhagens genéticas para a criação em sistema sem gaiolas, onde as aves consequentemente, não precisarão de adaptação.

\section{Agradecimentos}

Os autores agradecem à Fundação de Amparo à Pesquisa do Estado de São Paulo- FAPESP, Processo no 2017/14350-4 e também ao Conselho Nacional de Desenvolvimento Científico e Tecnológico CNPq, Processo ${ }^{\circ}$ 313339/2019-8 pelo suporte à pesquisa.

\section{Referências}

Ahammed, M., Chae, B. J., Lohakare, J., Keohavong, B., Lee, M. H., Lee, S. J., Kim, D. M., Lee, J. Y. \& Ohh S. J. (2014). Comparison of aviary, barn and conventional cage raising of chickens on laying performance and egg quality. Asian-Australasian Journal of Animal Science. 8(1), $1196-1203$.

Ali, A. B. A., Campbell, D. L. M., Karcher, D. M. \& Siegford, J. M. (2016). Influence of genetic strain and access to litter on spatial distribution of 4 strains of laying hens in an aviary system. Poultry Science. 95(11), 2489-2502.

Alves, S P., Silva, I. J. O. \& Piedade, S. M. (2007). Avaliação do bem-estar de aves poedeiras comerciais: efeitos do sistema de criação e do ambiente bioclimático sobre o desempenho das aves e a qualidade dos ovos. Revista Brasileira de Zootecnia, Viçosa, 36(1), $1388-1394$.

Barbosa Filho, J. A. D. (2004). Avaliação do bem-estar de aves poedeiras em diferentes sistemas de produção e condiçães ambientais, utilizando análise de imagem. 141 p. Dissertação (Dissertação em física do ambiente agrícola) - Escola Superior de Agricultura Luiz de Queiroz, Universidade São Paulo, Piracicaba.

Barbosa Filho, J. A. D., Silva, I. J. O., Silva, M. A. N. \& Silva, C. J. M. (2007). Avaliação dos comportamentos de aves poedeiras utilizando sequência de imagens. Engenharia Agrícola, Jaboticabal, 27 (1), 93-99.

Bari, M. S., Cohen, A. M. \& Campbell, D. L. M. (2020). Early rearing enrichments influenced nest use and egg quality in free-range laying hens. Animal. 14(6), 1249-1257.

Batista, E. S., Pereira, D. F., Sanchez, F. T., Guimarães, M. A., Nagai, D. K., Soares, N. M., Togashi, C. K. \& Bueno, L. G. (2012). Comportamento de uso do ninho e desempenho produtivo de poedeiras alojadas em diferentes densidades e tamanhos de grupo. Revista Educação Agrícola Superior, Brasília, 27(2), 119123 .

Baxter, M. R. (1994). The welfare problems of laying hens in battery cages. Vet. Rec. 134(1), 614-619.

Bayham, A. \& Özkan, S. (2010). Effects of a 16-hour light, 8-hour dark lighting schedule on behavioral traits and performance in male broiler chickens. Journal of Applied Poultry Research. 19(3), 263-273.

Belyavin, C. G. (1988). Egg quality as influenced by production systems. World's Poultry Science Journal, 44(1), 65-67.

Benyi, K., Norris, D. \& Tsatsinyane, P. M. (2006). Effect of stocking density and group size on the performance of White and brown Hyline layers in semiarid conditions. Tropical Animal Health Production, Heidelberg, 38(7-8), 619-624.

Bessei, W. (2006). Welfare of broilers: a review. World's Poultry Science Journal. 62(3), 455-466.

Bilcík, B., Keeling, L. J. IK. \& Keeling, L. J. (2000). Relationship between feather pecking in laying hens and the effect of group size. Applied Animal Behaviour Science, Amsterdam, 58(1), 55-66.

Bokkers, E. \& Koene, P. (2003). Behavior of fast- and slow growing broilers to 12 weeks of age and the physical consequences. Applied Animal Behavior Science. 81(1), 59-72. 
Brendler, C. \& Schrader, L. (2016). Perch use by laying hens in aviary systems. Applied Animal Behaviour Science. 182(1), 9-14.

Broom, D. M. (1988). The scientific assessment of animal welfare. Applied Animal Behavior Science, Amsterdam, 20(1), 5-19.

Broom, D. M. \& Molento, C. F. M. (2004). Bem-estar animal: Conceito e questões relacionadas-Revisão. Archives of Veterinary Science, 9(2), 1-11.

Casiraghi, E., Hidalgo, A. \& Rossi, M. (2005). Influence of weight grade on shell characteristics of marketed hen eggs. In: European Symposium on the Quality of Eggs and Egg Products, 10(1), 183-188.

Cherian, G., Langevin, C., Ajuyal, A., Lien, K. \& Sim, J.S. (1990). Research note: Effect of storage conditions and hard cooking on peelability and nutrient density of white and brown shelled eggs. Poultry Science, 69(1), 1614-1616.

Cooper, J. J. \& Appleby, M. C. (1995). Nesting behaviour of hens: Effects of experience on motivation. Applied Animal Behaviour Science. 42(4), $283-295$.

Cunningham, F. E., Cotteril, O. J. \& Funk, E. M. (1960). The effect of season and age of bird. I. On egg size, quality and yield. Poultry Science, 39(1), 289299.

Cunningham, D. L. \& Ostrander, C. E. (1982). The effects of strain and cage shape and density on performance and fearfulness of white leghorn layers. Poultry Science, Champaign, 61(2), 239-243.

D'eath, R. B. \& Keeling, L. J. (1999). Social discrimination and aggression by laying hens in large groups: from peck orders to social tolerance. Applied Animal Behaviour Science, 84(1), 197-212.

Dutra, D. R., Paschoalin, G. C., Souza, R. A., Mello, J. L. M. de, Giampietro-Ganeco, A., Ferrari, F. B., Souza, P. A., Borba, H., \& Pizzolante , C. C. (2021). Qualidade dos ovos frescos e armazenados em função do tempo de permanência nos ninhos em sistema cage-free. Research, Society and Development, 10(2), e39410211881. https://doi.org/10.33448/rsd-v10i2.11881

Eisen, E. J., Bohre, B. B. \& Mckean, H. E. (1962). The Haugh unit as a measure of egg albumen quality. Poultry Science, 41(1), 1461-1468.

Engel, J. M., Widowski, T. M., Tilbrook, A. J., Butler, K. L. \& Hemsworth, P. H. (2019). The effects of floor space and nest box access on the physiology and behavior of caged laying hens. Poultry Science. 98(2), 533-547.

Faure, J. M. \& Jones, R. B. (1982). Effects of age, access and time of day on perching behaviour in the domestic fowl. Applied Animal Ethology. 8(4), 357364.

Fletcher, D. L., Britton, W. M., Pesti, G. M. \& Rahn, A. P. (1983). The relationship of layer flock age and egg weight on egg component yields and solids content. Poultry Science, 62(1), 1800-1805.

Guo, Y. Y., Song, Z. G., Jiao, H. C., Song, Q. Q. \& Lin, H. (2012). The effect of group size and stocking density on the welfare and performance of hens housed in furnished cages during summer. Animal Welfare, 21(1), 41-49.

Haugh, R. R. (1937). The Haugh unit for measuring egg quality. United States Egg Poultry Magazine, 43(1), 552-555.

Heiman, V. \& Carver, J. S. (1936). The albumen index as a physical measurement of observed egg quality. Poultry Science, 15(1), 141-148.

Hidalgo, A., Rossi, M., Clerici, F. \& Ratti, S. (2008). A market study on the quality characteristics of eggs from different housing systems. Food Chemistry, London, 106(1), 1031-1038.

Holts, W. F. \& Almiquist, H. J. (1932). Measurement of deterioration in the stored hen's egg. United States Egg Poultry Magazine, $38(1), 70$.

Keeling, L. (1995). Spacing behaviour and an ethological approach to assessing optimum space allocations for groups of laying hens. Applied Animal Behaviour Science, 44(1), 171-186. doi: 10.1016/0168-1591(95)00612-V.

Lee, K. (1989). Laying performance and fear response of white leghorns as influenced by floor space allowance and group size. Poultry Science, Champaign, 68(10), 1333-36.

Li, G., Zhao, Y., Porter, Z. \& Purswell, J. L. (2021). Automated measurement of broiler stretching behaviors under four stocking densities via faster regionbased convolutional neural network. Animal. 15(1), Article 100059.

Liu, K., Xin, H., Shepherd, T. \& Zhao, Y. (2018). Perch-shape preference and perching behaviors of young laying hens. Applied Animal Behaviour Science. 203(1), 34-41.

Maclachlan, S. S., Ali, A. B. A., Toscano, M. J. \& Siegford, J. M. (2020). Influence of later exposure to perches and nests on flock level distribution of hens in an aviary system during lay. Poultry Science. 99(1), 30-38.

Nicol, C. J. (1987). Behaviour al responses of laying hens following a period of spatial restriction. Animal Behavior. 35(1), 1709-1719.

Nicol, C.J. (1989). Social influences on the comfort behavior of laying hens. Applied Animal Behaviour Science. 22(1), 75-81.

Onbasilar, E. E., Unal, N., Erdem, E., Kocakaya, A. \& Yaranoglu, B. (2015). Production performance, use of nest box, and external appearance of two strains of laying hens kept in conventional and enriched cages. Poultry Science, 94(4), 559-564.

Parsons, C. H. \& Mink, L. D. (1937). Correlation of methods for measuring the interior quality of eggs. United States Egg Poultry Magazine, 43(1), 484-489.

Pavlovski, Z., Hopic, S. \& Lukic, M. (2001). Housing system for layers and egg quality. Biotechnology Animal Husbandry, Belgare, 17(1), $197-201$. 
Research, Society and Development, v. 10, n. 4, e6010413833, 2021

(CC BY 4.0) | ISSN 2525-3409 | DOI: htttp://dx.doi.org/10.33448/rsd-v10i4.13833

Pereira, D. F., Batista, E. S., Sanches, F. T., Gabriel Filho, L. R. A. \& Bueno, L. G. F. (2013). Comportamento de poedeiras criadas a diferentes densidades e tamanhos de grupo em ambientes enriquecidos. Pesquisa Agropecuária Brasileira, Rio de Janeiro, 48(6), 682-688.

Pereira, C. L. (2007). Evaluation of thermal comfort and fibercement tiles on the performance of broilers confined in poultry houses with different types of rooting. 103 p. M. Sc. Dissertation - Faculdade de Zootecnia e Engenharia de Alimentos, Universidade de São Paulo, Pirassununga.

Proudfoot, F. G. (1962). The decline of internal egg quality during storage at $30^{\circ} \mathrm{F}$ and $70^{\circ} \mathrm{F}$ among six strains of Leghorns reared in confinement and on range. Poultry Science, 41(1), 98-103.

Rentsch, A. K., Rufener, C. B., Spadavecchia, C., Stratmann, A. \& Toscano, M. J. (2019). Laying hen's mobility is impaired by keel bone fractures and does not improve with paracetamol treatment. Applied Animal Behaviour Science. 216(1), 19-25.

Ribeiro, A. P., Silva, L. F., Menegali, I. \& Ferreira, F. (2020). Análise das variáveis ambientais e fisiológicas de aves poedeiras com e sem enriquecimento ambiental. Revista Brasileira de Engenharia de Biossistemas, 14(4), 412-418.

Rodenburg, T. B., Koene, P., Bokkers, E. A. M., Bos, M. E. H., Uitdehaag, K. A. \& Spruijt, B. M. (2005). Can short-term frustration facilitate feather pecking in laying hens? Applied Animal Behaviour Science, 91(1), 85-101. doi: 10.1016/j. applanim.2004.08.023.

Rodrigues, P. C. (1975). Contribuição ao estudo da conversão de ovos de casca branca e vermelha. Piracicaba. 57p. Dissertação (Mestrado) - Escola Superior de Agricultura “Luiz de Queiroz", Universidade de São Paulo.

Rossi, M. \& Pompei, C. (1995). Changes in some egg components and analytical values due to hen age. Poultry Science, 74(1), 152-160.

Silva, J. H. V., Albino, L. F. T. \& Godói, M. J. S. (2000). Efeito do extrato de urucum na pigmentação da gema dos ovos. Revista Brasileira de Zootecnia, 29(5), 1435-1439.

Silva, G. F., Pereira, D. F., Bueno, L. G. F., Santos, T. S. \& Tavares, B. O. (2013). Performance of laying hens and economic viability of different climatization systems. Italian Journal of Animal Science (Online), 12(1), 286-294. Retrieved from: https://www.tandfonline.com/doi/full/10.4081/ijas.2013.e47

Singer, P. (1991). Animal Liberation. 97-118. Retrieved from: https://link.springer.com/chapter/10.1007/978-1-349-25176-6_1

Singh, R., Cheng, K. M. \& Silversides, F. G. (2009). Production performance and egg quality of four strains of laying hens kept in conventional cages and floor pens. Poultry Science, Champaign, 88(1), 256-264.

Tavares, B. O., Pereira, D. F., Bueno, L. G. F. \& Silva, G. F. (2015). Behavior of Layers under Different Light Sources. Revista Brasileira de Ciência Avícola, 17(1), 511-516.

Trindade, J. L., Nascimento, J. W. B. \& Furtado, D. A. (2007). Qualidade do ovo de galinhas poedeiras criadas em galpões no semiárido paraibano. Revista Brasileira de Engenharia Agrícola Ambiental, 11(1), 652-657.

Tumova, E. \& Ebeid, T. (2003). Effect of housing system on performance and egg quality characteristics in laying hens. Scientia Agriculturae Bohemica, Prague, 34(1), 73-80.

Vits, A., Weitzenbürger, D., Hamann, H. \& Distl, O. (2005). Production, egg quality, bone strength, claw length, and keel bone deformities of laying hens housed in furnished cages with different group sizes. Poultry Science, Champaign, 84(1), 1511-1519.

Wilgus, H. S. \& Wagenen, A. V. (1936). The height of the firm albumen as a measure of its condition. Poultry Science, 15(1), 319-321. 